\title{
Computing Equilibria in Finance Economies
}

Citation for published version (APA):

Herings, P. J. J., \& Kubler, F. (2002). Computing Equilibria in Finance Economies. Mathematics of Operations Research, 27, 637-646. https://doi.org/10.1287/moor.27.4.637.296

Document status and date:

Published: 01/01/2002

DOI:

10.1287/moor.27.4.637.296

Document Version:

Publisher's PDF, also known as Version of record

\section{Please check the document version of this publication:}

- A submitted manuscript is the version of the article upon submission and before peer-review. There can be important differences between the submitted version and the official published version of record.

People interested in the research are advised to contact the author for the final version of the publication, or visit the DOI to the publisher's website.

- The final author version and the galley proof are versions of the publication after peer review.

- The final published version features the final layout of the paper including the volume, issue and page numbers.

Link to publication

\footnotetext{
General rights rights.

- You may freely distribute the URL identifying the publication in the public portal. please follow below link for the End User Agreement:

www.umlib.nl/taverne-license

Take down policy

If you believe that this document breaches copyright please contact us at:

repository@maastrichtuniversity.nl

providing details and we will investigate your claim.
}

Copyright and moral rights for the publications made accessible in the public portal are retained by the authors and/or other copyright owners and it is a condition of accessing publications that users recognise and abide by the legal requirements associated with these

- Users may download and print one copy of any publication from the public portal for the purpose of private study or research.

- You may not further distribute the material or use it for any profit-making activity or commercial gain

If the publication is distributed under the terms of Article $25 \mathrm{fa}$ of the Dutch Copyright Act, indicated by the "Taverne" license above, 
MATHEMATICS OF OPERATIONS RESEARCH

Vol. 27, No. 4, November 2002, pp. 637-646

Printed in U.S.A.

\title{
COMPUTING EQUILIBRIA IN FINANCE ECONOMIES
}

\author{
P. JEAN-JACQUES HERINGS AND FELIX KUBLER
}

\begin{abstract}
The general equilibrium model with incomplete asset markets is ideally suited for the study of problems in cross-sectional asset pricing and portfolio theory. In this paper, we develop a homotopy algorithm to approximate equilibria in these models. Since the algorithm is tailor made for so-called finance economies, the number of nonlinear equations that has to be solved for, and therefore the computing time, is an order of magnitude smaller than that of existing general-purpose algorithms. The algorithm is shown to be generically convergent. We implement the algorithm using HOMPACK. To illustrate its performance, we present various numerical examples and report running times.
\end{abstract}

1. Introduction. During the last two decades, there has been substantial interest in the general equilibrium model with incomplete asset markets, the GEI model. One of the important features of this model is its integrated approach to the real and financial sectors of an economy. For almost all GEI economies, a competitive equilibrium exists. The tools required to show existence of an equilibrium are demanding, and involve many results from differential topology, including the concept of the Grassmann manifold.

The complications of the GEI model imply that it is no longer possible to compute equilibria by the same methods that are used for the standard general equilibrium model. For instance, convergence of the algorithm of Scarf (1967) or the homotopy algorithm of Eaves (1972) is not guaranteed. By using algorithms that operate on the Grassmann manifold, Brown et al. (1996b) and DeMarzo and Eaves (1996) have produced computational methods that converge for a generic GEI economy. Brown et al. (1996a) develop a generically convergent algorithm by means of switching homotopies. This algorithm does not directly involve the Grassmann manifold, although its convergence proof does. For numerical purposes, one may also want to use the homotopy algorithm of Schmedders (1998) that does not involve homotopy switching. A drawback of that algorithm is that it is an open question whether as to it displays generic convergence to an equilibrium.

Our interest in computing solutions in the GEI model is derived from our desire to study the pricing of financial assets. For instance, to study whether the lessons of the capital asset pricing model remain valid in a setting with market incompleteness, heterogeneous investors, assets whose distributions have fat tails, and so on. For all these applications, one needs to approximate a continuous multivariate probability distribution by a finite probability distribution. In order to achieve a reasonable approximation, a big-state space is required. In a companion paper (Herings and Kubler 2000), we focus on the application to the capital asset pricing model and we need to compute equilibria for models with, for instance, $H=3$ agents and $J=8$ financial assets, and we need $S=32,768$ states of nature to approximate a multivariate lognormal distribution by a finite probability distribution.

All existing algorithms transform the equilibrium problem into a problem that involves so-called state prices. This is essential to show convergence but has the drawback that the

Received July 11, 2000; Revised March 21, 2001 and May 22, 2002.

MSC 2000 subject classification. Primary 91B28, 91B50, 50C30.

OR/MS subject classification. Primary: Finance/asset pricing.

Key words. Computational methods, asset pricing, general equilibrium, incomplete markets.

637

0364-765X/02/2704/0637/\$05.00

1526-5471 electronic ISSN, (C) 2002, INFORMS 
number of equations increases rapidly in the number of states. For instance, the homotopy proposed by Brown et al. (1996b) involves $2 S$ nonlinear equations. Another complication in applying that algorithm is that it involves closed-form solutions for the demand functions for assets of the agents, but such closed-form solutions are usually impossible to obtain when asset markets are incomplete. The solution to that problem is to state the problem not in terms of demand functions themselves but in terms of the first-order conditions of agents that yield the demand function. This approach is suggested in Garcia and Zangwill (1981) and is followed by Schmedders (1998). It increases the number of equations further, to $2 H S+(H-1) J+H$, so the use of Schmedders' (1998) algorithm to the models of the size $H=3, J=8$, and $S=32,768$ involves solving 196,627 nonlinear equations.

The algorithms of Brown et al. (1996b) and Schmedders (1998) are designed to deal with the general version of the GEI model. In many applications, one is interested in what is known as the finance version of the GEI model, or finance economy, for short. In the finance version of the GEI model, the modeling of the financial sector is the same as in the general version of the GEI model. The consumption sector, however, is drastically simplified, in that in each time period, at each state of the world, there is only one commodity, called income. In this paper, we develop an algorithm that is tailor made for finance economies. The restriction to finance economies leads to a great reduction in the number of equations to be solved for and, thereby, to strong improvements in computing times. If closed-form solutions for demand functions are available, then the number of nonlinear equations to be solved for by our algorithm equals $J-1$. Otherwise, we specify the first-order conditions and the number of nonlinear equations amounts to $(H+1)(J+1)-2$, which is 34 in the application reported on earlier.

Our algorithm is a homotopy algorithm. The use of deformations to solve nonlinear systems of equations dates back at least to Lahaye (1934). A constructive proof of Brouwer's fixed-point theorem for differentiable functions, based on a homotopy, was given in Kellogg et al. (1976). They use the nonretraction principle of Hirsch (1963) in their proof. We also exploit the differentiability that is present in the problem. We follow the system of differential equations as presented in Davidenko (1953) to follow the homotopy path in our implementation and show that this is possible to do for almost all finance economies. For recent surveys on homotopies, the reader is referred to Judd (1998) or Eaves and Schmedders (1999).

Compared to traditional general equilibrium theory, finance economies pose a number of additional difficulties. The prices of assets are not necessarily positive but may well be zero or negative, which rules out some of the algorithms that are used in traditional general equilibrium theory, for instance, the simplicial variable dimension algorithm of Doup et al. (1987) or its differentiable counterpart described in Herings (1997). Crucial to the convergence proof of homotopy methods applied to traditional general equilibrium models is the boundary behavior of the excess demand function. When prices of commodities converge to zero, demand for commodities explodes. As a price of zero has no special meaning in the case of financial assets, that boundary behavior cannot be used. The convergence proof of our homotopy algorithm builds on the approach to show existence of an equilibrium in finance economies as outlined in Hens (1991).

The paper is organized as follows. Section 2 introduces the notation and the model of a finance economy. In $\S 3$, we present an algorithm that is tailored to compute equilibria in finance economies. Special attention is given to the problem that closed-form solutions for demand functions of assets rarely exist in finance economies. A second, related algorithm is introduced that does not require closed-form solutions. In §4, we show generic convergence of the algorithm, that is, for an open set of finance economies with full Lebesgue measure, the algorithm converges to an equilibrium. Section 5 discusses the implementation of the algorithm, and in $\$ 6$, we describe numerical examples. Section 7 concludes. 
2. The finance economy. The finance version of the GE model describes an economy over two periods of time, $t=0,1$, with uncertainty over the state of nature resolving in period $t=1$. There are $S$ future states in the economy; at time $t=1$, one state of nature $s$ out of $S$ possible states of nature realizes. In each state $s=1, \ldots, S$, there is a single nondurable consumption good, which we call income.

There are $H$ agents, indexed by $h=1, \ldots, H$, that participate in the economy. Agent $h$ is characterized by the initial income stream $e^{h}=\left(e_{1}^{h}, \ldots, e_{S}^{h}\right)^{\top} \in \mathbb{R}_{++}^{S}$ and his preferences over income streams available for consumption $c^{h}=\left(c_{1}^{h}, \ldots, c_{S}^{h}\right)^{\top} \in \mathbb{R}_{++}^{S}$. Aggregate incomes are $e=\sum_{h=1}^{H} e^{h}$. The preferences of an agent are represented by a continuous, strictly quasiconcave utility function $u^{h}: \mathbb{R}_{++}^{S} \rightarrow \mathbb{R}$.

There are $J$ financial assets, indexed $j=1, \ldots, J$, that can be used to reshuffle income across states. Asset $j$ pays dividends at date $t=1$, denoted by $d^{j} \in \mathbb{R}^{S}$. The price of asset $j$ at time $t=0$ is $q_{j}$. We collect all assets' dividends in a payoff matrix

$$
A=\left(d^{1}, \ldots, d^{J}\right) \in \mathbb{R}^{S \times J} .
$$

At time $t=0$, agent $h$ chooses an asset portfolio $\theta^{h} \in \mathbb{R}^{J}$ satisfying $q \cdot \theta^{h}=0$. An asset portfolio consists of net trades by agent $h$ in each one of the assets. The requirement that $q \cdot \theta^{h}=0$ represents that the value of assets sold equals the value of assets bought. An asset portfolio $\theta^{h}$ uniquely defines the consumption of agent $h$ by $c^{h}=e^{h}+A \theta^{h}$. The net demand of agent $h, c^{h}-e^{h}$, therefore, belongs to the marketed subspace $\langle A\rangle=\left\{z \in \mathbb{R}^{S} \mid \exists \theta \in \mathbb{R}^{J}\right.$, $z=A \theta\}$.

The exogenous parameters defining a finance economy $\mathscr{E}=\left(\left(u^{h}, e^{h}\right)_{h=1, \ldots, H} ; A\right)$ are agents' utility functions and endowments, as well as the payoff matrix. Without loss of generality, we assume throughout that there are no redundant assets, $\operatorname{so} \operatorname{rank}(A)=J$. If there are redundant assets, it follows from an arbitrage argument that their price is uniquely determined by the prices of the other assets. Markets are incomplete when $J<S$. Prices for assets are said to be arbitrage free if it is not possible to achieve a nonnegative income stream in all states and a positive income stream in at least one state by trading in the available assets. It is well known that a price system $q \in \mathbb{R}^{J}$ precludes arbitrage if and only if there exists a strictly positive state price vector $\pi \in \mathbb{R}_{++}^{S}$ such that $q=\pi^{\top} A$.

Definition 2.1 (Competitive Equilibrium). A competitive equilibrium for an economy $\mathscr{E}$ is a collection of portfolio holdings $\theta^{*}=\left(\theta^{1 *}, \ldots, \theta^{H *}\right) \in \mathbb{R}^{H J}$ and prices for assets $q^{*} \in \mathbb{R}^{J}$ that satisfies the following conditions:

(1) $\theta^{h *} \in \arg \max _{\theta^{h} \in \mathbb{R}^{J}} u^{h}\left(c^{h}\right)$ s.t. $q \cdot \theta^{h}=0, c^{h}=e^{h}+A \theta^{h}$ and $c^{h} \in \mathbb{R}_{++}^{S}, h=1, \ldots, H$;

(2) $\sum_{h=1}^{H} \theta^{h *}=0$.

Under an additional assumption of strictly increasing utility functions, and a condition on the utility function, the so-called boundary condition presented in Assumption 1 below, existence of an equilibrium follows from the results of Geanakoplos and Polemarchakis (1986). Equilibrium prices for assets are always arbitrage free.

3. The algorithm. In this section, we develop a globally convergent algorithm to compute equilibria in finance economies. We strengthen the assumptions made so far by the Assumptions below, which state the standard assumptions on finance economies that are invoked when twice differentiability of the demand for assets is required.

Assumption 1. $u^{h}$ is three times continuously differentiable: $\partial u^{h}\left(c^{h}\right) \in \mathbb{R}_{++}^{S}$ for all $c^{h} \in$ $\mathbb{R}_{++}^{S}$ (strong monotonicity); $y^{\top} \partial^{2} u^{h}\left(c^{h}\right) y<0$ for all $y \neq 0$ such that $\partial u^{h}\left(c^{h}\right) y=0$, for all $c^{h} \in \mathbb{R}_{++}^{S}$ (negative Gaussian curvature); and $\left\{c^{h} \in \mathbb{R}_{++}^{S} \mid u^{h}\left(c^{h}\right) \geq u^{h}\left(\bar{c}^{h}\right)\right\}$ is closed in $\mathbb{R}^{S}$ for all $\bar{c}^{h} \in \mathbb{R}_{++}^{S}$ (boundary condition).

Assumption 2. $e^{h} \in \mathbb{R}_{++}^{S}$. 
Assumption 3. $\operatorname{rank}(A)=J$ and $A_{J}>0$. (The notation $x>0$ means that all components of the vector $x$ are nonnegative and at least one component is positive.)

Usually Assumption 3 is replaced by the weaker assumption that there is $\bar{\theta} \in \mathbb{R}^{J}$ such that $A \bar{\theta}>0$. Assumption 3 is without loss of generality. Indeed, if there is $\bar{\theta} \in \mathbb{R}^{J}$ such that $A \bar{\theta}>0$, we take $A \bar{\theta}$ as asset $J$ and we delete an asset $j$ for which $\bar{\theta}_{j} \neq 0$. Equilibria of the original economy are obtained by a simple transformation of the equilibria of the resulting economy. Under Assumption 3, it holds that $q_{J}>0$ for all arbitrage-free prices for assets $q$. It follows immediately that the solution to the optimization problem of an agent, as described in (1) of Definition 2.1, remains unchanged when all prices are multiplied by $\lambda>0$. We can therefore restrict ourselves to arbitrage-free asset prices for which $q_{J}=1$, and we define $Q=\left\{q \in \mathbb{R}^{J-1} \times\{1\} \mid \exists \pi \in \mathbb{R}_{++}^{S}, q=\pi^{\top} A\right\}$ to be the set of arbitrage-free prices for assets with this property.

Given arbitrage-free prices for assets $q \in Q$, the demand for assets by agent $h$, denoted $g^{h}(q)$, is the asset portfolio that solves the following maximization problem

$$
\max _{\theta^{h} \in \mathbb{R}^{J}} u^{h}\left(c^{h}\right) \text { s.t. } q \cdot \theta^{h}=0, c^{h}=e^{h}+A \theta^{h} \quad \text { and } \quad c^{h} \in \mathbb{R}_{++}^{S} .
$$

If prices for assets are arbitrage free, then the maximization problem is well defined. Assumption 1 guarantees that the solution to the optimization problem is unique.

From the demand function for assets of agent $h, g^{h}: Q \rightarrow \mathbb{R}^{J}$, the total demand function for assets $G: Q \rightarrow \mathbb{R}^{J}$ follows as $G=\sum_{h=1}^{H} g^{h}$. Prices for assets $q^{*}$ induce a competitive equilibrium for an economy $\mathscr{E}$ if and only if $G\left(q^{*}\right)=0$.

The following properties are useful when showing convergence of the algorithm.

Lemma 3.1. If the economy $\mathscr{E}$ satisfies Assumptions 1-3, then the following properties hold:

1. The function $G: Q \rightarrow \mathbb{R}^{J}$ is twice continuously differentiable.

2. For all $q \in Q, q \cdot G(q)=0$.

3. If $\left(q^{n}\right)_{n \in \mathbb{N}}$ is a sequence in $Q$ converging to $\bar{q} \in \partial Q$ or diverging, that is, $\left\|q^{n}\right\|_{2} \rightarrow \infty$, then for all $\tilde{q} \in Q, \tilde{q} \cdot G\left(q^{n}\right) \rightarrow \infty$. ( $\partial Q$ represents the relative boundary of $\left.Q.\right)$

Proof. See Hens (1991).

Our algorithm starts from some initial price system $q^{0} \in Q$. We will address the issue of choosing a sensible $q^{0}$ in $\S 5$. A price system $q$ with the last component deleted is denoted by $\hat{q}$; in particular, $\hat{q}^{0}$ specifies the first $J-1$ components of the initial price system $q^{0}$. The function $G$ with the last component deleted is denoted by $\widehat{G}$. We propose to compute equilibria in a finance economy by means of the homotopy $\mathscr{H}:[0,1] \times Q \rightarrow \mathbb{R}^{J-1}$ defined by

$$
\mathscr{H}(t, q)=t \widehat{G}(q)+(1-t)\left(\hat{q}^{0}-\hat{q}\right) .
$$

We are looking for solutions to $\mathscr{H}(t, q)=0$. If $\mathscr{H}(1, q)=0$ then; since $q_{J}=1$, Lemma 3.1.2 implies that $G_{J}(q)=0$. It follows that $q$ is a competitive equilibrium price system.

4. Generic convergence. A homotopy is in general constructed in such a way that there is a unique solution to $\mathscr{H}(0, q)=0$; solutions to $\mathscr{H}(1, q)=0$ are solutions to the problem of interest; and the unique solution to $\mathscr{H}(0, q)=0$ is linked by a path of solutions to $\mathscr{H}(t, q)=0$, for varying $t$, to one solution to $\mathscr{H}(1, q)=0$. By following this path, a solution to the problem of interest is found. When the unique solution to $\mathscr{H}(0, q)=0$ is indeed linked by a path to a solution to $\mathscr{H}(1, q)=0$, then the homotopy is said to converge. For an excellent discussion on the numerical techniques available to follow the path we refer to Allgower and Georg (1990).

It cannot always be guaranteed that our homotopy converges. There may exist economies such that the set of solutions $\mathscr{H}^{-1}(0)$ does not link the unique solution to $\mathscr{H}(0, q)=0$ 
to one solution to $\mathscr{H}(1, q)=0$. The set $\mathscr{H}^{-1}(0)$ may display bifurcations and even higher dimensional solution sets. Nevertheless, we show that for typical economies, convergence of the homotopy takes place, and nonconvergence can only happen in exceptional cases.

To make precise what typical means, we have to parameterize economies. We fix a tuple of utility functions $\left(u^{h}\right)_{h=1, \ldots, H}$ and an asset payoff matrix $A$. Then any choice of initial endowments $\left(e^{h}\right)_{h=1, \ldots, H} \in \mathbb{R}_{++}^{H S}$ induces an economy $\mathscr{E}=\left(\left(u^{h}, e^{h}\right)_{h=1, \ldots, H} ; A\right)$. In this way, economies are parametrized by initial endowments. A property is said to be typical if it holds for a class of economies that is large in both a topological and a measure-theoretic sense, that is, when it holds for a set of initial endowments that is open and of full Lebesgue measure.

THEOREM 4.1. Fix utility functions $\left(u^{h}\right)_{h=1, \ldots, H}$ and an asset payoff matrix A satisfying Assumptions 1 and 3. Then, for all initial endowments $e$ in an open set of initial endowments with full Lebesgue measure $E \subset \mathbb{R}_{++}^{H S}$, the homotopy $\mathscr{H}$ related to the resulting economy $\mathscr{E}$ satisfies the following:

1. $\mathscr{H}^{-1}(\{0\})$ is a compact $C^{2}$ one-dimensional manifold with boundary, with the boundary given by $\mathscr{H}^{-1}(\{0\}) \cap(\{0,1\} \times Q)$.

2. There is an odd number of solutions in $\mathscr{H}^{-1}(\{0\}) \cap(\{1\} \times Q)$, i.e., there is an odd number of competitive equilibria.

For any choice of initial endowments $e \in \mathbb{R}_{++}^{H S}$, the homotopy $\mathscr{H}$ related to the resulting economy $\mathscr{E}$ satisfies the following:

3. There is one solution in $\mathscr{H}^{-1}(\{0\}) \cap(\{0\} \times Q)$.

4. There is no sequence $\left(t^{n}, q^{n}\right)_{n \in \mathbb{N}}$ in $\mathscr{H}^{-1}(\{0\})$ converging to $(t, q) \in[0,1] \times \partial Q$, or diverging, $\left\|\left(t^{n}, q^{n}\right)\right\|_{2} \rightarrow \infty$.

Proof. The only solution in $\mathscr{H}^{-1}(\{0\}) \cap(\{0\} \times Q)$ is obviously given by $\left(0, q^{0}\right)$, which proves 4.1.3.

Suppose $\left(t^{n}, q^{n}\right)_{n \in \mathbb{N}}$ is a sequence in $\mathscr{H}^{-1}(\{0\})$ converging to $(\bar{t}, \bar{q}) \in[0,1] \times \partial Q$ or a diverging sequence. Then, $t^{n} \widehat{G}\left(q^{n}\right)+\left(1-t^{n}\right)\left(\hat{q}^{0}-\hat{q}^{n}\right)=0$, so, for $\bar{q} \in Q$,

$$
\begin{aligned}
0 & =\left(\hat{q}^{0}-\hat{q}^{n}\right) \cdot\left(t^{n} \widehat{G}\left(q^{n}\right)+\left(1-t^{n}\right)\left(\hat{q}^{0}-\hat{q}^{n}\right)\right) \\
& =t^{n} \hat{q}^{0} \cdot \widehat{G}\left(q^{n}\right)-t^{n} \hat{q}^{n} \cdot \widehat{G}\left(q^{n}\right)+\left(1-t^{n}\right)\left(\hat{q}^{0}-\hat{q}^{n}\right) \cdot\left(\hat{q}^{0}-\hat{q}^{n}\right) \\
& =t^{n} \hat{q}^{0} \cdot \widehat{G}\left(q^{n}\right)+t^{n} G_{J}\left(q^{n}\right)+\left(1-t^{n}\right)\left(\hat{q}^{0}-\hat{q}^{n}\right) \cdot\left(\hat{q}^{0}-\hat{q}^{n}\right) \\
& =t^{n} q^{0} \cdot G\left(q^{n}\right)+\left(1-t^{n}\right)\left(\hat{q}^{0}-\hat{q}^{n}\right) \cdot\left(\hat{q}^{0}-\hat{q}^{n}\right) .
\end{aligned}
$$

By Lemma 3.1.3, it follows that

$$
q^{0} \cdot G\left(q^{n}\right) \rightarrow \infty
$$

It is also clear that $\left(\hat{q}^{0}-\hat{q}^{n}\right) \cdot\left(\hat{q}^{0}-\hat{q}^{n}\right)>0$ for $n$ sufficiently large. As a consequence, the right-hand side of Equation (1) is strictly positive for $n$ sufficiently large, a contradiction. Solutions to the homotopy equations stay away from $[0,1] \times \partial Q$ and are not diverging, which shows 4.1.4.

To show 4.1.1, notice that the continuity of $\mathscr{H}$ yields that $\mathscr{H}^{-1}(\{0\})$ is closed in $[0,1] \times Q$. Since, moreover, solutions to the homotopy equations stay away from $[0,1] \times \partial Q$ and are not diverging, it follows that $\mathscr{H}^{-1}(\{0\})$ is compact.

The proof of 4.1 .1 is completed by showing that $\partial_{\hat{q}} \mathscr{H}(0, q)$ and, generic in initial endowments, $\partial_{\hat{q}} \mathscr{H}(1, q)$ and $\partial_{t, \hat{q}} \mathscr{H}(t, q)$ have full rank for points in $\mathscr{H}^{-1}(\{0\})$.

A simple calculation reveals that

$$
\partial_{\hat{q}} \mathscr{H}(0, \bar{q})=-I,
$$

where $I$ is a $(J-1)$-dimensional identity matrix, so $\partial_{\hat{q}} \mathscr{H}(0, q)$ has full rank $J-1$. 
We define $\overline{\mathscr{H}}:(0,1) \times Q \times \mathbb{R}_{++}^{S} \rightarrow \mathbb{R}^{J-1}$ by

$$
t \widehat{G}\left(q, e^{1}\right)+(1-t)\left(\hat{q}^{0}-\hat{q}\right),
$$

where $\widehat{G}\left(q, e^{1}\right)=\hat{g}^{1}\left(q, e^{1}\right)+\sum_{h=2}^{H} \hat{g}^{h}(q)$. We show next that $\overline{\mathscr{H}}$ is transversal to zero, or equivalently, that $\partial_{t, \hat{q}, e^{1}} \overline{\mathscr{H}}\left(\bar{t}, \bar{q}, \bar{e}^{-1}\right)$ has full row rank whenever $\overline{\mathscr{H}}\left(\bar{t}, \bar{q}, \bar{e}^{-1}\right)=0$.

For $j^{\prime}=1, \ldots, J-1$, define the asset portfolio $\bar{\theta}^{j^{\prime}}$ by $\bar{\theta}_{J}^{j^{\prime}}=-\bar{q}_{j^{\prime}}, \bar{\theta}_{j^{\prime}}^{j^{\prime}}=1$, and $\bar{\theta}_{j}^{j^{\prime}}=0$, $j \neq J, j \neq j^{\prime}$. Then changing the initial endowment of agent 1 to $\bar{e}^{-1}+\alpha A \bar{\theta}^{j^{\prime}}$ with $\alpha$ sufficiently small, changes his asset demand to $g^{1}\left(\bar{q}, \bar{e}^{-1}\right)-\alpha \bar{\theta}^{j^{\prime}}$. Since the vectors $\bar{\theta}^{j^{\prime}}, j^{\prime}=$ $1, \ldots, J-1$, are independent, even with component $J$ deleted, it follows that $\partial_{e^{1}} \widehat{G}\left(\bar{q}, \bar{e}^{-1}\right)$ has rank $J-1$. From this, it is immediate that $\partial_{\hat{q}, e} \overline{\mathscr{H}}\left(\bar{t}, \bar{q}, \bar{e}^{1}\right)$ has rank $J-1$. By the Transversality Theorem, see Mas-Colell (1985, I.2.2); the set of economies for which $\partial_{t, \hat{q}} \mathscr{H}(\bar{t}, \bar{q})$ has full rank for all points in $\mathscr{H}^{-1}(\{0\})$ has full Lebesgue measure.

Exactly the same argument shows that for a set of initial endowments with full Lebesgue measure, $\partial_{\hat{q}} \mathscr{H}(1, \bar{q})$ has full rank for points in $\mathscr{H}^{-1}(\{0\}) \cap(\{1\} \times Q)$.

The transversality proofs show that for a set of initial endowments with full Lebesgue measure, $\mathscr{H}^{-1}(\{0\})$ is a $C^{2}$ one-dimensional manifold with boundary, where the boundary is given by $\mathscr{H}^{-1}(\{0\}) \cap(\{0,1\} \times Q)$. It follows by a standard argument that the set of initial endowments for which transversality holds can be taken open and of full Lebesgue measure, which completes the proof of 4.1.1.

For an open set of initial endowments with full Lebesgue measure, $\mathscr{H}^{-1}(\{0\})$ is a compact $C^{2}$ one-dimensional manifold with boundary and is therefore a finite collection of arcs and loops. (An arc is a set homeomorphic to the unit interval and a loop a set homeomorphic to the unit circle.) Each arc has two boundary points. Since all boundary points belong to $\{0,1\} \times Q$, and there is exactly one boundary point in $\{0\} \times Q$, it follows that for an open set of initial endowments with full Lebesgue measure, there is an odd number of solutions in $\mathscr{H}^{-1}(\{0\}) \cap(\{1\} \times Q)$, which shows 4.1.2.

Since $\mathscr{H}$ is a system of $J-1$ independent equations in $J$ variables, it is not surprising that $\mathscr{H}^{-1}(\{0\})$ is generically a compact one-dimensional manifold with boundary, i.e., a finite collection of arcs and loops. There is a unique solution to $\mathscr{H}(0, q)=0$. The boundary behavior of $G$ guarantees that there is no sequence $\left(t^{n}, q^{n}\right)_{n \in \mathbb{N}}$ in $\mathscr{H}^{-1}(\{0\})$ converging to $(t, q) \in[0,1] \times \partial Q$ or diverging. Therefore, the unique solution to $\mathscr{H}(0, q)=0$ is generically part of a path in $\mathscr{H}^{-1}(\{0\})$ that does not run off to the boundary but reaches $t=1$. The unique solution to $\mathscr{H}(0, q)=0$ is thereby connected to exactly one point $\left(1, q^{*}\right) \in \mathscr{H}^{-1}(\{0\})$, a competitive equilibrium for $\mathscr{E}$, and the homotopy converges. Notice that there is no need to compute the set $Q$ explicitly.

Corollary 4.2. Let $\mathscr{E}$ be an economy satisfying Assumptions 1-3. Then, for an open set of initial endowments with full Lebesgue measure, the homotopy $\mathscr{H}$ converges to a competitive equilibrium.

If there are multiple equilibria, then in addition to the arc connecting $q^{0}$ and a competitive equilibrium $q^{*}$, there is a finite number of arcs, each one having two more competitive equilibria as its end points. This gives a constructive proof that there is an odd number of competitive equilibria. In fact, using the properties of a homotopy, we can get an index theorem for our economy, a result already obtained by Hens (1991), and for certain classes of economies with more than one good per state by Schmedders (1998).

The computation of the demand for assets as a function of prices for assets is not necessarily an easy problem. The theoretical homotopy $\mathscr{H}$ is therefore replaced by the diffeomorphic implementable homotopy $\mathscr{H}^{*}:[0,1] \times Q \times \mathbb{R}^{H(J-1)} \times \mathbb{R}^{H} \rightarrow \mathbb{R}^{(H+1)(J+1)-2}$,

$$
\mathscr{H}^{*}(t, q, \theta, \lambda)=\left\{\begin{array}{l}
t \sum_{h=1}^{H} \theta_{j}^{h}+(1-t)\left(q_{j}^{0}-q_{j}\right), \quad j=1, \ldots, J-1, \\
\partial u^{h}\left(e^{h}+A \theta^{h}\right) A-\lambda^{h} q^{\top}, \quad h=1, \ldots H, \\
q \cdot \theta^{h}, \quad h=1, \ldots, H .
\end{array}\right.
$$


We have replaced the demand functions of the agents by their first-order conditions, an approach proposed in Garcia and Zangwill (1981).

THEOREM 4.3. Let $\mathscr{E}$ be an economy satisfying Assumption $1-3$. Then $\mathscr{H}^{*^{-1}}(\{0\})$ is $C^{2}$ diffeomorphic to $\mathscr{H}^{-1}(\{0\})$.

Proof. The sets $\mathscr{H}^{*^{-1}}(\{0\})$ and $\mathscr{H}^{-1}(\{0\})$ are $C^{2}$ diffeomorphic if there exists a $C^{2}$ diffeomorphism $f: \mathscr{H}^{*^{-1}}(\{0\}) \rightarrow \mathscr{H}^{-1}(\{0\})$, so $f$ is injective, surjective, and both $f$ and $f^{-1}$ are twice continuously differentiable.

It holds that $(\bar{t}, \bar{q}, \bar{\theta}, \bar{\lambda}) \in \mathscr{H}^{*^{-1}}(\{0\})$ if and only if $(\bar{t}, \bar{q}) \in \mathscr{H}^{-1}(\{0\}), \bar{\theta}^{h}=g^{h}(\bar{q}), h=$ $1, \ldots, H$, and $\bar{\lambda}^{h}=\partial u^{h}\left(e^{h}+A^{h}(\bar{q})\right) A_{J} / \bar{q}_{J}, h=1, \ldots, H$.

Consider $f: \mathscr{H}^{*^{-1}}(\{0\}) \rightarrow \mathscr{H}^{-1}(\{0\})$ defined by $f(\bar{t}, \bar{q}, \bar{\theta}, \bar{\lambda})=(\bar{t}, \bar{q})$ and $f^{-1}: \mathscr{H}^{-1}(\{0\})$ $\rightarrow \mathscr{H}^{*^{-1}}(\{0\})$ defined by

$$
f^{-1}(\bar{t}, \bar{q})=\left(\bar{t}, \bar{q}, g^{1}(\bar{q}), \ldots, g^{H}(\bar{q}), \partial u^{1}\left(e^{1}+A g^{1}(\bar{q})\right) \frac{A_{\cdot J}}{\bar{q}_{J}}, \ldots, \partial u^{H}\left(e^{H}+A g^{H}(\bar{q})\right) \frac{A_{\cdot J}}{\bar{q}_{J}}\right) .
$$

It follows from the previous paragraph that $f$ is injective and surjective. It is obvious that $f$ is twice continuously differentiable and that the twice continuous differentiability of $f^{-1}$ follows from the twice continuous differentiability of $g^{h}$ and $\partial u^{h}$.

Since $\mathscr{H}^{*^{-1}}(\{0\})$ is diffeomorphic to $\mathscr{H}^{-1}(\{0\})$, the results of Theorem 4.1 carry over to $\mathscr{H}^{*^{-1}}(\{0\})$.

COROLlary 4.4. Let $\mathscr{E}$ be an economy satisfying Assumptions 1-3. Then, for an open set of initial endowments with full Lebesgue measure, the homotopy $\mathscr{H}^{*}$ converges to a competitive equilibrium.

5. Implementation. The speed of homotopy algorithms depends mainly on two factors, the number of equations and the arc length of the homotopy path. A quick comparison shows the great benefits of developing a special purpose homotopy tailored to the finance GEI model. The homotopy algorithms as reported in Brown et al. (1996a) and Schmedders (1998) are designed to deal with the general GEI model with multiple commodities per state but can be applied to finance economies.

The homotopy proposed by Brown et al. (1996a) needs closed-form solutions for excess demand functions and should therefore be compared with our homotopy $\mathscr{H}$. Applied to twoperiod finance economies, their algorithm has $2 S$ equations, whereas ours only has $J-1$. The algorithm of Schmedders (1998) does not require closed-form solutions for excess demand functions and also uses the first-order conditions. The number of equations of his algorithm amounts to $2 H S+(H-1)(J+1)+1$, whereas the number of equations in our algorithm $\mathscr{H}^{*}$ equals $(H+1)(J+1)-2$.

In both cases, we roughly need a fraction $J / 2 S$ only of the equations of alternative algorithms. This is especially favorable when $S$ is high, as is, for instance, the case in any application where a continuous multivariate distribution of endowments and asset returns in Period 1 has to be approximated accurately by a discrete one. A specific example concerns our companion paper (Herings and Kubler 2000), where we need $S=32,768$ states of nature to reasonably approximate a continuous multivariate distribution of the endowments of three agents and eight financial assets.

On top of the great number of equations saved, our method also has the flexibility of choosing the initial price system as desired, contrary to the homotopies of Brown et al. (1996a) or Schmedders (1998). Since it is not too hard to make a reasonable guess for an equilibrium price system, our algorithm will generally cause a substantial reduction in the arc length of the homotopy path. Suppose that agent $h$ is the only agent in the economy. It follows immediately from the system of first-order conditions that the competitive equilibrium price system is given by $q^{*}=\pi^{h} A$, where the so-called state price vector $\pi^{h}$ is equal 
TABLE 1. Running times (mm.ss)

\begin{tabular}{lcccc}
\hline & 10,000 States & 20,000 States & 30,000 States & 40,000 States \\
\hline 2 agents, 5 assets & 0.31 & 0.58 & 2.09 & 3.17 \\
2 agents, 8 assets & 1.31 & 3.15 & 5.28 & 7.00 \\
3 agents, 5 assets & 1.02 & 2.59 & 4.12 & 6.49 \\
3 agents, 8 assets & 2.01 & 5.21 & 8.14 & 10.11 \\
\hline
\end{tabular}

to $\partial u^{h}\left(e^{h}\right) / \partial u^{h}\left(e^{h}\right) A_{. J}$. A reasonable guess for $q^{0}$ is therefore obtained by computing $\pi^{0} A$, where $\pi^{0}$ is the average over all the agents' $\pi^{h}=\partial u^{h}\left(e^{h}\right) / \partial u^{h}\left(e^{h}\right) A_{J}$.

We implemented the algorithm using HOMPACK—a suite of FORTRAN 77 subroutines designed to solve systems of nonlinear homotopy equations with path-following methods. See Watson (1979) and Watson et al. (1987) for details on HOMPACK.

6. Numerical examples. In order to illustrate the performance of our algorithm and to show how running times increase with the number of agents, the number of assets, and the number of states, we consider several examples. In all examples, agents have constant relative risk aversion utility of the form

$$
u^{h}\left(c^{h}\right)=\sum_{s=1}^{S} \frac{1}{S} v^{h}\left(c_{s}^{h}\right), \quad c^{h} \in \mathbb{R}_{++}^{S},
$$

with

$$
v^{h}\left(c_{s}^{h}\right)=\frac{\left(c_{s}^{h}\right)^{1-\gamma^{h}}}{1-\gamma^{h}}, \quad c_{s}^{h}>0
$$

where $\gamma^{h}$ is the coefficient of relative risk aversion.

We set $\gamma^{1}=2, \gamma^{2}=4$ and $\gamma^{3}=6$. As long as coefficients of relative risk aversion stay relatively small (below 8), the actual choice of these coefficients has only small effects on running times.

In order to examine how running times change with the number of agents, the number of assets and the number of states, we consider the following specifications: An example with two agents and five assets, an example with two agents and eight assets, an example with three agents and five assets, and an example with three agents and eight assets. For each example, we consider the case of 10,000 states, 20,000 states, 30,000 states and 40,000 states.

In order to specify state contingent asset payoffs and individual endowments, we generate random numbers. We take individual endowments to be drawn from a uniform distribution on $[1,10]$ and dividends to be drawn from a uniform distribution on [0.1, 5].

For each of the 16 examples we compute equilibria for 100 different draws, and we report average running times.

Table 1 shows the running times in minutes. All running times refer to an implementation in FORTRAN 77 on a 500-MHz Pentium III processor running Red Hat Linux.

Since we subsitute for market clearing, we obtain that solving for an equilibrium involves solving a system of $H(J+1)$ nonlinear system. The number of equations is independent of the number of states. Note that the running times do increase significantly with the number of states, since the time needed for a single evaluation of the first-order conditions increases. The increase is more or less linear in the number of states. If the number of states would increase the number of equations, running times in this order of magnitude would be impossible. 
A central idea underlying our homotopy algorithm is that it is not necessarily desirable to have a high precision along the path. This is consistent with the general idea underlying HOMPACK. Watson et al. (1987) point out that tracking the homotopy path is "merely a means to an end"- - finding the solution to $H(\cdot, 1)=0$ - and that no computational effort should be wasted following the path too closely. However, if one sets the error tolerance along the path incorrectly (e.g., too large), the algorithm is likely to lose the right path. In Table 1 , the relative tracking tolerance is set to $5 \cdot 10^{-5}$. HOMPACK allows the user to independently set the relative error tolerance at the solution, for the table we set this tolerance to $10^{-9}$. While the effect of different error tolerances at the solution on running times is not significant, the relative tracking tolerance influences error times substantially. If this error tolerance is set to $10^{-3}$ the algorithm does not converge for all examples considered. When it does converge (approximately $80 \%$ of all draws), running times are reduced by a factor of 1.5 . If the error is set to a value of $10^{-7}$, average running times increase by a factor of 2 .

7. Conclusion. In this paper, we develop a homotopy algorithm to compute equilibria in the finance version of the GEI model that is particularly useful for cases with a large state space. The generic convergence of this algorithm is shown, where "generic" means that for an open set of finance economies with full Lebesgue measure, convergence takes place. The implementation of the algorithm is discussed. Its effectiveness is verified by means of numerical examples. In Herings and Kubler (2000), the algorithm is used to explore asset pricing implications of the GEI model when the number of states is large.

Acknowledgments. The research of Jean-Jacques Herings has been made possible by a fellowship of the Royal Netherlands Academy of Arts and Sciences and a grant from the Netherlands Organization for Scientific Research (NWO). While this paper was being written, this author enjoyed the generous hospitality of the Cowles Foundation for Research in Economics at Yale University and of CORE at Université Catholique de Louvain. Felix Kubler gratefully acknowledges the financial support of an Anderson Dissertation Fellowship.

\section{References}

Allgower, E. L., K. Georg. 1990. Numerical Continuation Methods: An Introduction. Springer Verlag, New York. Brown, D. J., P. M. DeMarzo, B. C. Eaves. 1996a. Computing equilibria when asset markets are incomplete. Econometrica 64 1-27.

1996b. Computing zeros of sections of vector bundles using homotopies and relocalization. Math. Oper. Res. 21 26-43.

Davidenko, D. 1953. On a new method of numerical solution of systems of nonlinear equations. Doklady Akad. Nauk USSR 88 601-602.

DeMarzo, P. M., B. C. Eaves. 1996. Computing equilibria of GEI by relocalization on a Grassmann manifold. $J$. Math. Econom. 26 479-497.

Doup, T. M., G. van der Laan, A. J. J. Talman. 1987. The $\left(2^{n+1}-2\right)$-ray algorithm: A new simplicial algorithm to compute economic equilibria. Math. Programming 39 241-252.

Eaves, B. C. 1972. Homotopies for computation of fixed points. Math. Programming 3 1-22.

, K. Schmedders. 1999. General equilibrium models and homotopy methods. J. Econom. Dynam. Control 23 1249-1279.

Garcia, C., W. Zangwill. 1981. Pathways to Solutions, Fixed Points, and Equilibria. Prentice Hall, Englewood Cliffs, NJ.

Geanakoplos, J. D., H. M. Polemarchakis. 1986. Existence, regularity, and constrained suboptimality of competitive allocations when the asset market is incomplete. W. P. Heller, R. M. Starr, D. A. Starrett, eds. Uncertainty, Information and Communication: Essays in Honor of K. J. Arrow, Vol. III. Cambridge University Press, Cambridge, U.K., 65-96.

Hens, T. 1991. Structure of general equilibrium models with incomplete markets. Doctoral dissertation, University of Bonn.

Herings, P. J. J. 1997. A globally and universally stable price adjustment process. J. Math. Econom. 27 163-193.

Herings, P. J. J., F. Kubler. 2000. The robustness of CAPM-A computational approach. METEOR Research Memorandum 00/02, University of Maastricht, Maastricht, The Netherlands, 1-34. 
Hirsch, M. W. 1963. A proof of the nonretractibility of a cell onto its boundary. Proc. Amer. Math. Soc. 14 364-365.

Judd, K. L. 1998. Numerical Methods in Economics. MIT Press, Cambridge, U.K.

Kellogg, R. B., T. Y. Li, J. Yorke. 1976. A constructive proof of the Brouwer fixed-point theorem and computational results. SIAM J. Numer. Anal. 13 473-483.

Lahaye, E. 1934. Une méthode de résolution d'une catégorie d'équations transcendantes. C. R. Acad. Sci. Paris 198 1840-1842.

Mas-Colell, A. 1985. The Theory of General Economic Equilibrium: A Differentiable Approach. Cambridge University Press, Cambridge, U.K., 45.

Scarf, H. 1967. The approximation of fixed points of a continuous mapping. SIAM J. Appl. Math. 15 1328-1343.

Schmedders, K. 1998. Computing equilibria in the general equilibrium model with incomplete asset markets. $J$. Econom. Dynam. Control 22 1375-1401.

Watson, L. T. 1979. A globally convergent algorithm for computing fixed points of C2 maps. Appl. Math. Comput. 5 297-311.

, S. C. Billups, A. P. Morgan. 1987. HOMPACK: A suite of codes for globally convergent homotopy algorithms. ACM Trans. Math. Software 13 281-310.

P. J. J. Herings: Department of Economics, Universiteit Maastricht, PO Box 616, 6200 MD Maastricht, The Netherlands; e-mail: p.herings@algec.unimaas.nl

F. Kubler, Department of Economics, Stanford University, Stanford, California 94305-6072; e-mail: fkubler@stanford.edu 
Copyright 2002, by INFORMS, all rights reserved. Copyright of Mathematics of Operations Research is the property of INFORMS: Institute for Operations Research and its content may not be copied or emailed to multiple sites or posted to a listserv without the copyright holder's express written permission. However, users may print, download, or email articles for individual use. 
Copyright 2002, by INFORMS, all rights reserved. Copyright of Mathematics of Operations Research is the property of INFORMS: Institute for Operations Research and its content may not be copied or emailed to multiple sites or posted to a listserv without the copyright holder's express written permission. However, users may print, download, or email articles for individual use. 